\title{
双铁电复合材料的制备及其光电化学性能研究
}

\author{
张亚萍 ${ }^{1}$, 雷宇轩 ${ }^{1}$, 丁文明 ${ }^{2}$, 于濂清 ${ }^{2}$, 朱帅霏 ${ }^{1}$ \\ (1. 中国石油大学(华东)1. 理学院; 2. 材料科学与工程学院, 青岛 266580)
}

摘 要: 通过模板法制备钒酸铋 $\left(\mathrm{BiVO}_{4}\right)$ 薄膜, 用溶胶一凝胶法制备铁电材料铁酸铋 $\left(\mathrm{BiFeO}_{3}\right)$ 并对 $\mathrm{BiVO}_{4}$ 进行修饰, 以半导体复合的方式提高 $\mathrm{BiVO}_{4}$ 的光电化学性能。电化学测试结果表明, 经 $\mathrm{BiFeO}_{3}$ 修饰后, $\mathrm{BiVO}_{4}$ 薄膜的光电化学 性能有所提高, 其中经 $\mathrm{BiFeO}_{3}$ 旋涂 5 次后的 $\mathrm{BiVO}_{4}$ 薄膜具有最优的光电化学性能, 光电流密度达到 $0.72 \mathrm{~mA} \cdot \mathrm{cm}^{-2}$, 较未修饰样品提高了 $67.4 \%$ 。利用外场极化调节能带弯曲可以显著地提高 $\mathrm{BiVO}_{4} / n \mathrm{BiFeO}_{3}$ 铁电复合物的光电化学性 能, 复合物经正极化 $20 \mathrm{~V}$ 电压处理后的光电流密度最高为 $0.91 \mathrm{~mA} \cdot \mathrm{cm}^{-2}$, 比 $\mathrm{BiVO}_{4}$ 薄膜提升了 1 倍以上, 具有良 好的光电化学性能。 $\mathrm{BiFeO}_{3}$ 与 $\mathrm{BiVO}_{4}$ 复合后有利于形成异质结, 促进光生电子、光生空穴的产生与分离, 并且外 场极化调节能带弯曲使光生电荷加速转移, 是铁电复合物光电化学性能提高的主要原因。

关 键 词: $\mathrm{BiVO}_{4} ; \mathrm{BiFeO}_{3}$; 铁电复合材料; 光电化学性能

中图分类号: TQ174 文献标识码: A

\section{Preparation and Photoelectrochemical Property of the Dual-ferroelectric Composited Material}

\author{
ZHANG Yaping ${ }^{1}$, LEI Yuxuan ${ }^{1}$, DING Wenming ${ }^{2}$, YU Lianqing ${ }^{2}$, ZHU Shuaifei ${ }^{1}$
}

(1. College of Science, China University of Petroleum, Qingdao 266580, China; 2. College of Material Science and Engineering, China University of Petroleum, Qingdao 266580, China)

\begin{abstract}
BiVO}_{4}$ film was synthesized via template method, the ferroelectric material $\mathrm{BiFeO}_{3}$ was prepared by Sol-Gel method to modify $\mathrm{BiVO}_{4}$. By means of dual-ferroelectric semiconductor composition, the photochemical properties of $\mathrm{BiVO}_{4}$ was greatly improved. The electrochemical test results show that the superior photoelectrochemical properties of $\mathrm{BiVO}_{4}$ film are achieved after spin-coating with $\mathrm{BiFeO}_{3}$ for 5 times. It owns an optimal photocurrent density of $0.72 \mathrm{~mA} \cdot \mathrm{cm}^{-2}$, which is $67.4 \%$ higher than that of pure $\mathrm{BiVO}_{4}$. The energy band bending regulation via electric field polarization could further boost the photoelectrochemical property of $\mathrm{BiVO}_{4} / n \mathrm{BiFeO}_{3}$ ferroelectric composite. The highest photocurrent density of the composite after polarization at $20 \mathrm{~V}$ reaches $0.91 \mathrm{~mA} \cdot \mathrm{cm}^{-2}$, which is more than twice of pure $\mathrm{BiVO}_{4}$ film. The combination of $\mathrm{BiFeO}_{3}$ and $\mathrm{BiVO}_{4}$ is favorable for forming heterojuncting, generating and separating of photogenic electrons and holes. The electric field polarization regulating band bending accelerates the photogenic charge transfer, which is the main reason for the improved photoelectrochemical properties of ferroelectric composite.
\end{abstract}

Key words: $\mathrm{BiVO}_{4} ; \mathrm{BiFeO}_{3}$; ferroelectric composite material; photoelectrochemical property

收稿日期: 2019-10-11; 收到修改稿日期：2020-01-11

基金项目: 国家自然科学基金(21476262); 青岛市科技发展计划(14-2-4-108-jch)

National Natural Science Foundation of China (21476262); Technology Project of Qingdao (14-2-4-108-jch)

作者简介: 张亚萍(1967-), 女, 博士, 副教授. E-mail: zhangyp@upc.edu.cn

ZHANG Yaping(1967-), female, PhD, associate professor. E-mail: zhangyp@upc.edu.cn

通讯作者：于濂清，教授. E-mail: iyy2000@163.com

YU Lianqing, professor. E-mail: iyy2000@163.com 
作为一种光电化学性能优异的 $\mathrm{n}$ 型半导体, $\mathrm{BiVO}_{4}$ 由于特有的电子分布结构与特殊的晶体结构, 在可见光区域内具有良好的光吸收特性与稳定性, 在光解水、光催化降解有机污染物、太阳能电池电 极等方面具有重要的应用价值与研究前景 ${ }^{[1-2]}$ 。 $\mathrm{BiVO}_{4}$ 主要以四方锆石矿、单斜白铇矿、四方白铇 矿三种晶型存在, 其中单斜白铇矿晶型的 $\mathrm{BiVO}_{4}$ 的 禁带宽度较窄 $(2.4 \mathrm{eV})$, 具有较好的光吸收特性 ${ }^{[3]}$ 。 目前, $\mathrm{BiVO}_{4}$ 的制备方法主要有固相反应法、离子热 合成法、水热法、化学共沉积法等 ${ }^{[4]}$, Zhou 等 ${ }^{[5]}$ 通过 超声法制备了平均粒径为 $50 \mathrm{~nm}$ 的单斜白铇相 $\mathrm{BiVO}_{4}$ 纳米颗粒，具有良好的光吸收性能。 $\mathrm{Yu}$ 等 ${ }^{[6]}$ 以澳化十六烷基三甲铵 (CTAB)为微反应器, 通过水 热法制备的 $\mathrm{BiVO}_{4}$ 纳米纤维具有良好的单斜白铇相 晶体结构。 $\mathrm{BiVO}_{4}$ 由于表面缺陷和较差的析氧反应 (OER)动力学 ${ }^{[7]}$, 光催化反应过程中受光激发产生 的光生载流子易复合, 造成光电流密度降低, 进而 导致光电转化效率不佳。

$\mathrm{BiFeO}_{3}$ 作为一种典型的钙钛矿材料, 具有较小 的禁带宽度 $\left(E_{\mathrm{g}}=2.2 \sim 2.7 \mathrm{eV}\right)$, 是一种第三代光催化 剂之一。且由于其良好的铁电性能, $\mathrm{BiFeO}_{3}$ 自发极 化产生的内禀电场能够加速电荷的传输, 导致能带 弯曲, 可以有效抑制光生载流子的复合 ${ }^{[8]}$, 因此常 利用其构建复合材料体系以提高光生电子与空穴的 分离效率与光电流密度, 提高材料的光电化学性 能。Lee 等 ${ }^{[9]}$ 通过激光脉冲沉积法用 $\mathrm{BiFeO}_{3}$ 对 $\mathrm{TiO}_{2}$ 纳米管(TNT)进行修饰, 产物在 $0.2 \mathrm{~V}(v s . \mathrm{Ag} / \mathrm{AgCl})$ 的光电流密度达到 $0.54 \mathrm{~mA} \cdot \mathrm{cm}^{-2}$, 较 TNT 提高了一 倍。Wang 等 ${ }^{[10]}$ 通过旋涂法与光沉积法构建 $\mathrm{Co}-\mathrm{Pi} / \mathrm{BiFeO}_{3}$ 共催化体系, 将复合物在 $0 \mathrm{~V}$ (vs. $\mathrm{Ag} / \mathrm{AgCl}$ ) 的光电流密度提升至 $0.05 \mathrm{~mA} \cdot \mathrm{cm}^{-2}$, 比 $\mathrm{BiFeO}_{3}$ 提高了 8 倍。

本工作以半导体复合的方式, 使用 $\mathrm{BiFeO}_{3}$ 对 $\mathrm{BiVO}_{4}$ 进行修饰, 制备双铁电复合材料, 以扩大其 光响应范围, 有效抑制光生载流子复合, 通过协同 效应提高光电化学性能 ${ }^{[11]}$, 并且进一步探究外场极 化对其光电化学性能的影响。研究结果对提高铋基 铁电复合材料的光电化学性能与光催化性能有借鉴 意义。

\section{1 实验方法}

\section{$1.1 \mathrm{BiVO}_{4} / \mathrm{BiFeO}_{3}$ 复合材料的制备}

将导电玻璃( $\mathrm{FTO}$ ) 作为工作电极在三电极体系 下进行电沉积, 制备 BiOI $(-0.1 \mathrm{~V}, 10 \mathrm{~min})$, 以
$0.4 \mathrm{~mol} \cdot \mathrm{L}^{-1}$ 的 $\mathrm{KI}$ 溶液(硝酸调节 $\mathrm{pH}$ 至 1.7 ) 为溶剂 配制 $0.04 \mathrm{~mol} \cdot \mathrm{L}^{-1}$ 的 $\mathrm{Bi}\left(\mathrm{NO}_{3}\right)_{3} \cdot 5 \mathrm{H}_{2} \mathrm{O}$ 溶液，与 $0.23 \mathrm{~mol} \cdot \mathrm{L}^{-1}$ 对苯醌乙醇溶液以体积比 $5: 2$ 充分混 合后作为电沉积液。将乙酰丙酮氧钒 $\left(\mathrm{VO}(\mathrm{acac})_{2}\right)$ 溶 于二甲亚砜中 $\left(0.2 \mathrm{~mol} \cdot \mathrm{L}^{-1}\right)$, 随后将该溶液滴加到制 备的 $\mathrm{BiOI}$ 上, 于 $450{ }^{\circ} \mathrm{C}$ 保温 $2 \mathrm{~h}$ (升温速度 $2{ }^{\circ} \mathrm{C} / \mathrm{min}$ )。 最后使用 $1 \mathrm{~mol} \cdot \mathrm{L}^{-1} \mathrm{NaOH}$ 溶液漂洗后得到 $\mathrm{BiVO}_{4}$ (简称 BVO)。

将 $0.85 \mathrm{~g} \mathrm{Bi}\left(\mathrm{NO}_{3}\right)_{3} \cdot 5 \mathrm{H}_{2} \mathrm{O} 、 0.8 \mathrm{~g} \mathrm{Fe}\left(\mathrm{NO}_{3}\right)_{3} \cdot 9 \mathrm{H}_{2} \mathrm{O}$ 、 $3.32 \mathrm{~g}$ 柠檬酸、 $4 \mathrm{~g}$ 乙二醇混合并加热摚拌后, 在 FTO 上旋涂 $(2300 \mathrm{r} / \mathrm{min}, 60 \mathrm{~s})$ 。随后在管式炉中 $500{ }^{\circ} \mathrm{C}$ 下保温 $30 \mathrm{~min}$ (升温速度 $5{ }^{\circ} \mathrm{C} / \mathrm{min}$ ) 得到纯 $\mathrm{BiFeO}_{3}$ (简称 $\mathrm{BFO}$ )。 $\mathrm{BiVO}_{4} / \mathrm{BiFeO}_{3}$ 复合物的制备示 意图如图 1 所示。在 $\mathrm{BiVO}_{4}$ 上分别旋涂 1 7 次 $\mathrm{BiFeO}_{3}$, 所得样品标记为 $\mathrm{BVO} / n \mathrm{BFO}(n=1,3,5,7)$ 。

\section{$1.2 \mathrm{BVO} / \mathrm{nBFO}$ 复合物形貌和性能测试}

采用 X 射线衍射仪(XRD, DX-2700, 中国丹东 方圆, $\mathrm{Cu}-\mathrm{K} \alpha$ 辐射, $\lambda=0.15418 \mathrm{~nm}, 40 \mathrm{kV}, 30 \mathrm{~mA}$ )进 行物相分析; 采用场发射扫描电子显微镜(SEM) (S-4800, 日本日立公司)进行形貌和能谱(EDS)表征; 使用拉曼光谱仪(DXR Microscope, Thermo Fisher) 进行光谱分析; 采用上海辰华电化学工作站 $(\mathrm{CHI}$ $760 \mathrm{E}$ ) 进行光电化学性能测试, $\mathrm{Pt}$ 丝为辅助电极, 待 测样品为工作电极, 饱和 $\mathrm{Ag} / \mathrm{AgCl}$ 作为参比电极, 电解液为 $1 \mathrm{~mol} \cdot \mathrm{L}^{-1}$ 的 $\mathrm{Na}_{2} \mathrm{SO}_{3}$ 溶液, 氙灯提供模拟 太阳光光源 $\left(\mathrm{AM} 1.5 \mathrm{G}\right.$, 功率密度 $100 \mathrm{~mW} \cdot \mathrm{cm}^{-2}$, 波 长范围 380 780 nm), 电化学阻抗图谱(EIS)扫描范 围为 $0.01 \sim 10^{5} \mathrm{~Hz}$; 外场极化使用 $\mathrm{BVO} / 5 \mathrm{BFO}$ 作为工 作电极, 施加 $\pm 20 \mathrm{~V}$ 电压(FTO 为对电极, 极化 $20 \mathrm{~min}$ ); 采用光致发光荧光光谱仪(PL) (SOFN 7-PLSpec I, 浙江)和紫外-可见吸收光谱仪(UV-Vis) (U-3900, 日本)进行光性能表征。
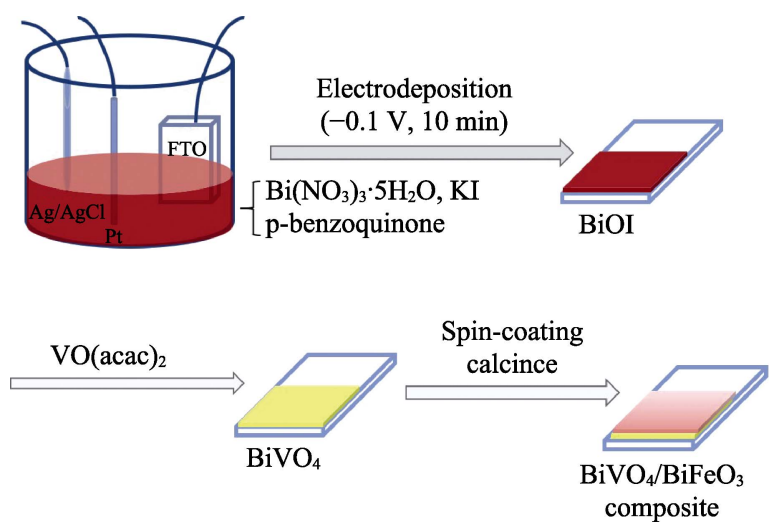

图 $1 \mathrm{BVO} / n \mathrm{BFO}$ 复合物的制备示意图

Fig. 1 Schematic diagram for preparation of $\mathrm{BVO} / n \mathrm{BFO}$ composite 


\section{2 结果与讨论}

\subsection{XRD 测试}

$\mathrm{BVO} / 5 \mathrm{BFO}$ 复合物与未修饰试样的 XRD 图谱 如图 2 所示, BVO 特征衍射峰与斜钒铋矿 (PDF\#14$0688)$ 相符合，在 $2 \theta=28.94^{\circ} 、 46.03^{\circ}$ 等位置分别出现 了其(121)、(042)等晶面的特征峰。样品在 $2 \theta=22.49^{\circ}$ 、 $32.13^{\circ}$ 等位置出现的衍射峰, 分别与 BFO (PDF\#200169)的(101)、(110)等晶面位置相一致。由此表明, 制备的 $\mathrm{BVO} / 5 \mathrm{BFO}$ 复合物的结晶度较好。

\subsection{SEM 分析}

由图 3(a)可知，制备的 BiOI 呈鳞片状，长度为 $1 \sim 2 \mu \mathrm{m}$ 。由图 3(b)可以看出, BVO 纳米颗粒分布均 匀, 表面呈多孔状, 孔隙的形成可能是由于溶剂的 疏水性以及㷽烧时溶剂挥发所致。由于二甲亚砜 (DMSO) 具有较弱的疏水性, 能够与 BiOI 表面充分 接触, 在加热过程中 DMSO 挥发, 从而形成三维多 孔结构(如图 3(c)所示), 可以提供更多的活性中心, 进而提高样品的催化活性 ${ }^{[11-12]}$ 。由图 3(d) 可知, $\mathrm{BVO} / 5 \mathrm{BFO}$ 复合物薄膜厚度约 $100 \mu \mathrm{m}$ 。结合 XRD 图谱与 EDS 图像(图 3(e h)) 可知, 制备的 BVO/5BFO 复合物分布均匀。

\section{3 拉曼光谱分析}

图 4 为 $\mathrm{BVO} / 5 \mathrm{BFO}$ 与未修饰试样的拉曼光谱 图。BVO/5BFO 与 BVO 分别在 $124 、 209 、 324 、 366$ 、 709 和 $825 \mathrm{~cm}^{-1}$ 处出现 $\mathrm{BiVO}_{4}$ 的振动峰, 与斜钒铋 矿的特征峰相一致。其中 $209 \mathrm{~cm}^{-1}$ 处的振动峰来自 $\mathrm{VO}_{4}$ 基团中 $\mathrm{V}-\mathrm{O}$ 键的自旋和频移的外模; 324 和 $366 \mathrm{~cm}^{-1}$ 处的振动峰分别来自 $\mathrm{VO}_{4}$ 基团中 $\mathrm{V}-\mathrm{O}$ 的反 对称弯曲模 $\left(\mathrm{B}_{\mathrm{g}}\right)$ 和对称弯曲模 $\left(\mathrm{A}_{\mathrm{g}}\right) ; 709 \mathrm{~cm}^{-1}$ 处较弱 的拉曼峰来自 $\mathrm{V}-\mathrm{O}$ 键的反对称弯曲模 $\left(\mathrm{A}_{\mathrm{g}}\right), 825 \mathrm{~cm}^{-1}$ 处的振动峰来自 $\mathrm{V}-\mathrm{O}$ 键的对称伸缩模 $\left(\mathrm{A}_{\mathrm{g}}\right)^{[13]}$ 。经 $\mathrm{BiFeO}_{3}$ 修饰的 $\mathrm{BiVO}_{4}$ 的振动峰位置未发生改变, 表 明复合前后 $\mathrm{BiVO}_{4}$ 的晶体结构未发生改变 ${ }^{[14]}$ 。此外,

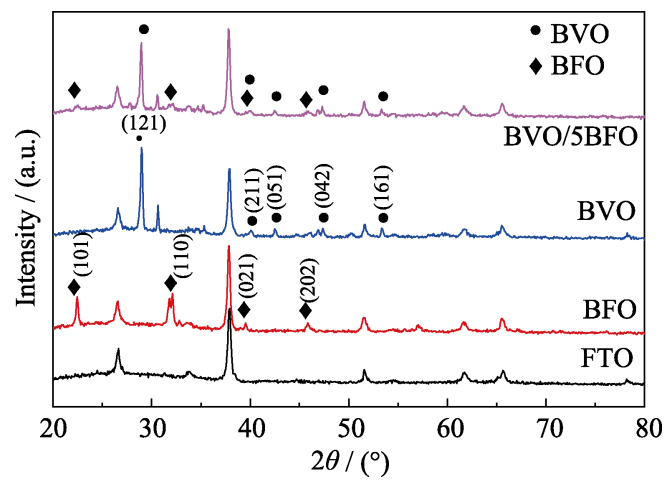

图 $2 \mathrm{BVO} / 5 \mathrm{BFO} 、 \mathrm{BVO} 、 \mathrm{BFO} 、 \mathrm{FTO}$ 的 XRD 图谱

Fig. 2 XRD patterns of BVO/5BFO, BVO, BFO, and FTO
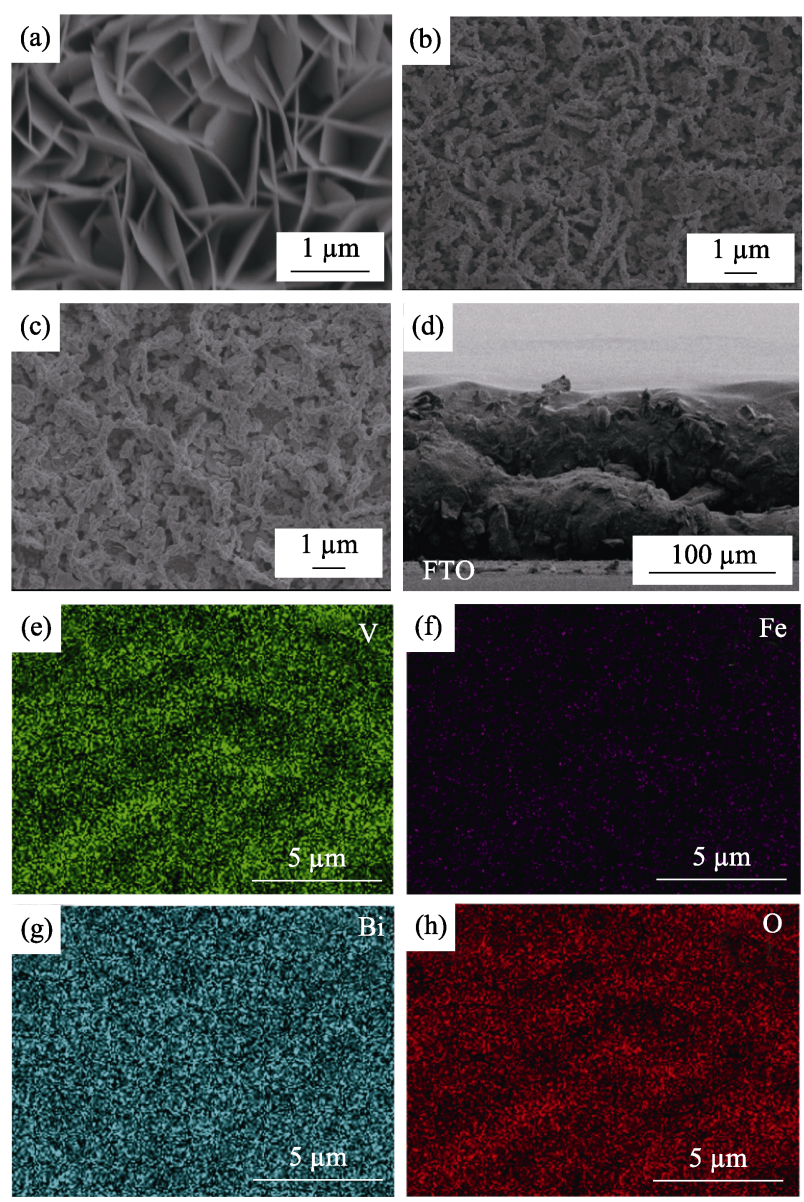

图 3 (a) BiOI、(b) BVO、(c) BVO/5BFO 的表面 SEM 照片; $\mathrm{BVO} / 5 \mathrm{BFO}$ 的(d)截面 SEM 照片和(e h) EDS mapping 图像 Fig. 3 Surface SEM images of (a) BiOI, (b) BVO, (c) BVO/ 5BFO, and (d) Cross-sectional SEM image and (e-h) EDS mapping images of $\mathrm{BVO} / 5 \mathrm{BFO}$

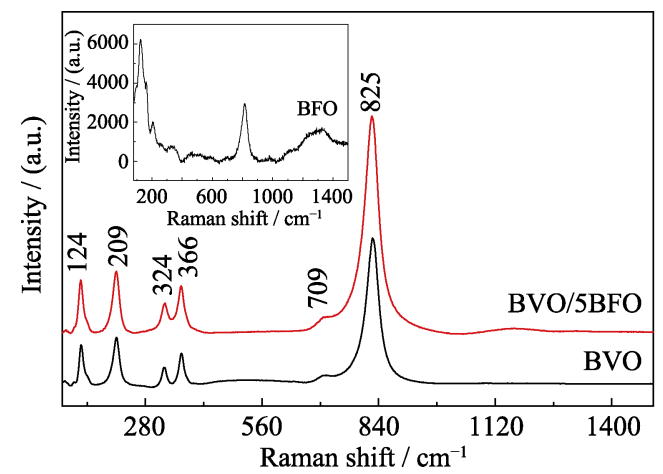

图 $4 \mathrm{BVO} / 5 \mathrm{BFO} 、 \mathrm{BVO} 、 \mathrm{BFO}$ 的拉曼光谱图

Fig. 4 Raman spectra of $\mathrm{BVO} / 5 \mathrm{BFO}, \mathrm{BVO}$ and $\mathrm{BFO}$

$\mathrm{BVO} / 5 \mathrm{BFO}$ 复合物的特征峰强度高于 $\mathrm{BVO}$, 可能是 $\mathrm{BFO}$ 的特征峰位置与 BVO 基本重合所导致的。

\section{4 光致发光光谱图与紫外-可见光谱图分析}

图 5(a)为制备样品的光致发光光谱图, 由图可 见，BVO 中的光生载流子复合较多，说明有效电荷 浓度较低, 光生载流子寿命较短。经 $\mathrm{BFO}$ 修饰后 $\mathrm{BVO}$ 中的光生载流子被有效地分离，因此复合较 

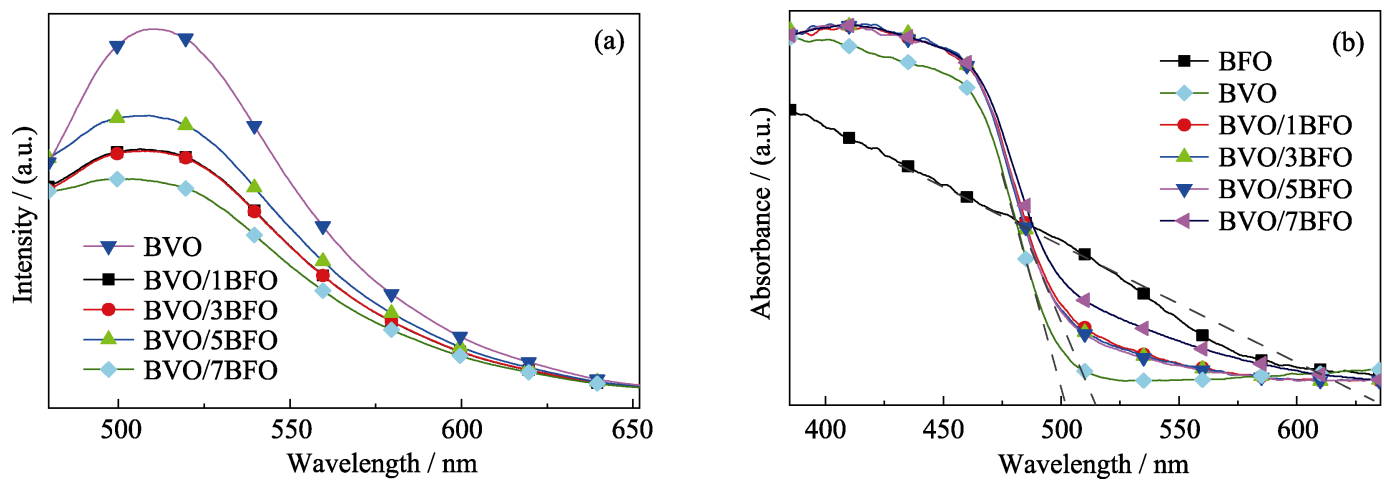

图 $5 \mathrm{BFO} 、 \mathrm{BVO}$ 和 $\mathrm{BVO} / n \mathrm{BFO}$ 的(a)光致发光光谱图和(b)紫外-可见吸收光谱图

Fig. 5 (a) PL and (b) UV-Vis spectra of $\mathrm{BFO}, \mathrm{BVO}$ and $\mathrm{BVO} / n \mathrm{BFO}$

少。图 5(b)为各样品的紫外-可见吸收光谱图, 可以 表征材料的光吸收性能并计算其禁带宽度。

BFO 修饰的 BVO 薄膜的吸收带边均发生了红 移, 在可见光区域内的光吸收能力增强, 进而提高 了可见光利用率 ${ }^{[15]}$ 。这是因为 $\mathrm{BiFeO}_{3}$ 具有较窄的禁 带宽度, 使得吸光范围有所拓宽。对紫外-可见吸收 光谱图中谱线的线性部分作切线, 取切线与横轴的 交点为吸收带边 $\lambda$, 通过公式 $E_{\mathrm{g}}=1240 / \lambda$ 可以得到半 导体的禁带宽度 $\left(E_{\mathrm{g}}\right)$ 。图 5(b)中, 纯 BVO 的吸收带 边大约为 $499 \mathrm{~nm}$, 计算可得其禁带宽度 $E_{\mathrm{g}}$ 约为 $2.5 \mathrm{eV}$, 而经修饰后的 $\mathrm{BVO} / 5 \mathrm{BFO}$ 禁带宽度 $E_{\mathrm{g}}$ 约为 $2.41 \mathrm{eV}$ 。

\section{$2.5 \mathrm{BVO} / \mathrm{nBFO}$ 光电化学性能分析}

图 6(a)为外加偏压为 $0 \mathrm{~V}(v s . \mathrm{Ag} / \mathrm{AgCl})$ 时各样 品的 $I-t$ 曲线。在模拟太阳光照状态改变时, 不同旋
涂层数下制备的 $\mathrm{BVO} / n \mathrm{BFO}$ 复合物均表现出灵敏 的光电信号响应，电流可以迅速达到稳定值，复合 物的光响应电流密度随着旋涂层数增加呈现先增大 后减小的趋势, 其中 $\mathrm{BVO} / 5 \mathrm{BFO}$ 的光响应电流密度 最大，达到 $0.72 \mathrm{~mA} \cdot \mathrm{cm}^{-2}$, 超过 $\mathrm{BVO}$ 薄膜 $\left(0.43 \mathrm{~mA} \cdot \mathrm{cm}^{-2}\right)$ 和 $\mathrm{BFO}\left(0.006 \mathrm{~mA} \cdot \mathrm{cm}^{-2}\right)$ 。经 $\mathrm{BFO}$ 修 饰后, 复合物的光电流密度比未经修饰的 BVO 提 高了 $67.4 \%$ 。这主要是因为复合物体系有利于光生 电子、光生空穴的产生与分离, $\mathrm{BiFeO}_{3}$ 修饰可以拓 宽光吸收范围, 受光激发时产生更多的光生电子一 空穴对，并且 $\mathrm{BiVO}_{4}$ 与 $\mathrm{BiFeO}_{3}$ 界面处形成的异质结 能够有效抑制光生载流子复合。 $\mathrm{BVO} / 7 \mathrm{BFO}$ 的瞬态 光电流密度为 $0.33 \mathrm{~mA} \cdot \mathrm{cm}^{-2}$, 低于 $B V O$, 可能是因 为旋涂层数过多, $\mathrm{BVO}$ 表面覆盖了过量的 $\mathrm{BFO}$, 一 定程度上阻碍了 $\mathrm{BVO}$ 薄膜的光吸收, 导致光生电
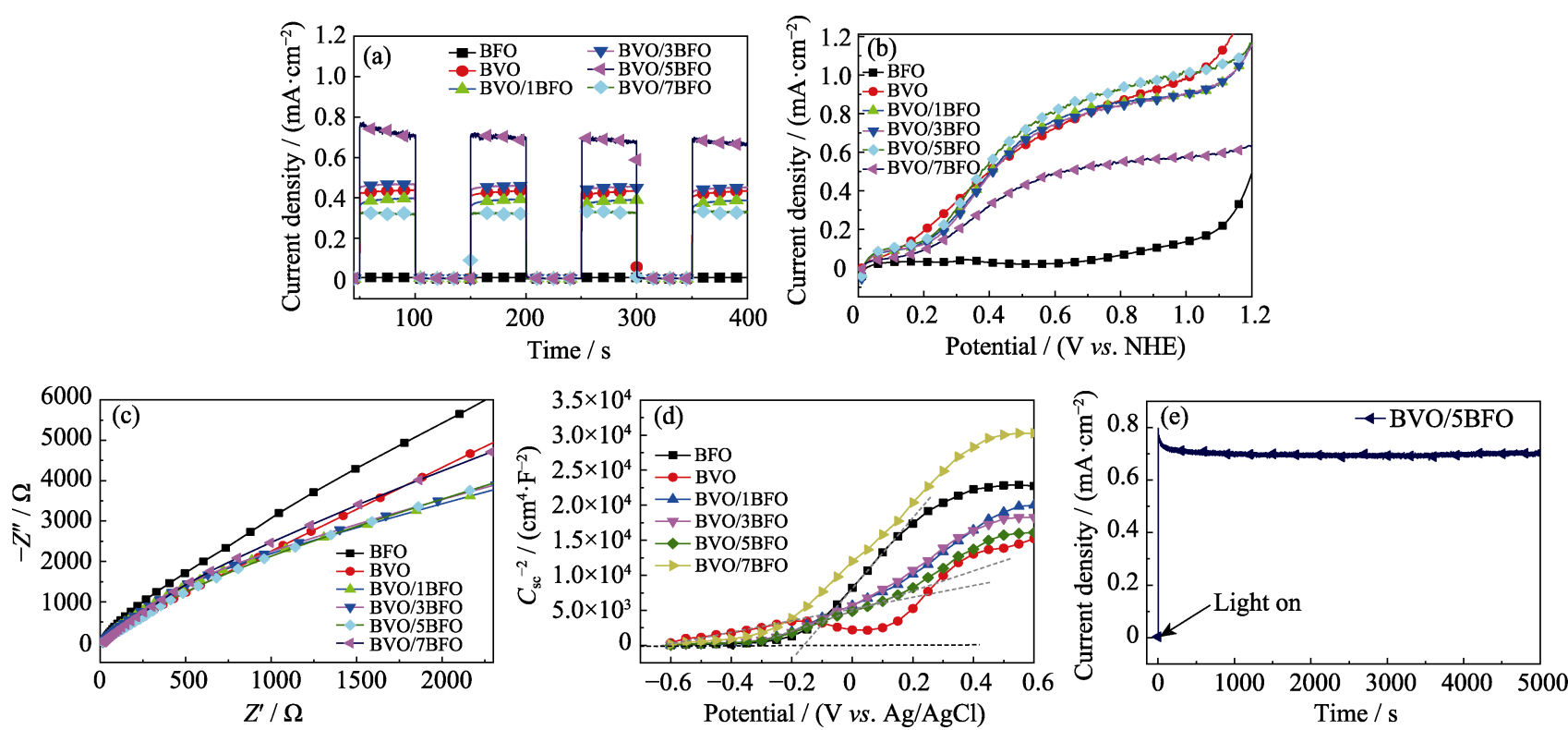

图 $6 \mathrm{BVO}, \mathrm{BFO}, \mathrm{BVO} / n \mathrm{BFO}$ 的(a) $I-t$ 曲线、(b)线性伏安曲线、(c)电化学阻抗谱图、

(d)莫特-肖特基曲线; (e)BVO/5BFO 的循环稳定性曲线

Fig. 6 (a) I-t curves, (b) linear sweep voltammograms, (c) Nyquist plots, (d) Mott-Schottky plots of BFO, $\mathrm{BVO}$, and $\mathrm{BVO} / n \mathrm{BFO}$, and (e) recycling performance of $\mathrm{BVO} / 5 \mathrm{BFO}$ 
子和空穴浓度下降 ${ }^{[16]}$ 。

图 6(b)为各样品的线性伏安曲线, 在可见光照 射下，经修饰的 $\mathrm{BiVO}_{4}$ 光电流密度随外加电压的增 加而增大，且增幅大于纯 $\mathrm{BiVO}_{4}$ 。在外加偏压 $0.6 \mathrm{~V}$ (vs. $\mathrm{NHE}$ )处, BVO/5BFO 的电流密度达到 $0.824 \mathrm{~mA} \cdot \mathrm{cm}^{-2}$, 比 $\mathrm{BiVO}_{4}\left(0.738 \mathrm{~mA} \cdot \mathrm{cm}^{-2}\right)$ 提高 $11.6 \%$ ，这可以归因 于经 $\mathrm{BiFeO}_{3}$ 修饰后 $\mathrm{BiVO}_{4}$ 的表面活性增加, 并且 $\mathrm{BiFeO}_{3}$ 的极化效应导致能带弯曲, 加速光生电荷转 移，降低光生电子-空穴的复合几率，从而有效地提 高了载流子的浓度。

图 6(c)为各样品的电化学阻抗图谱(EIS), 图中 $\mathrm{BVO} / 5 \mathrm{BFO}$ 的圆弧半径小于 $\mathrm{BVO}$ 的圆弧半径, 说 明其阻抗相对较小, 更有利于载流子的传输 ${ }^{[17]}$ 。

$\mathrm{BVO} / n \mathrm{BFO}(n=1,3,7)$ 的阻抗大于 $\mathrm{BVO}$ 而小于 $\mathrm{BFO}$, 主要是因为新相的引入增加了表面缺陷的数量, 导 致阻抗增大。图 6(d)为样品的莫特-肖特基曲线, 由 电荷载流子浓度 $N_{\mathrm{D}}=2\left(\mathrm{e} \varepsilon \varepsilon_{0} m\right)^{-1}$ (其中, $\varepsilon_{0} 、 e 、 \varepsilon$ 为 常数, $m$ 为莫特-肖特基曲线线性部分的斜率)可知, 电荷载流子浓度与莫特-肖特基曲线的线性部分的 斜率成反比。随着旋涂层数的增加, $\mathrm{BVO} / n \mathrm{BFO}$ 复 合物中的载流子浓度先升高后降低, 其中 $\mathrm{BVO} / 5 \mathrm{BFO}$ 的载流子浓度最大, 这与 $I-t$ 曲线中 $\mathrm{BVO} / 5 \mathrm{BFO}$ 光电流密度的结果一致。此外, 根据莫 特-肖特基曲线可以计算出 $\mathrm{BiVO}_{4}$ 与 $\mathrm{BiFeO}_{3}$ 的平带 电位分别为 -0.6 与 $-0.15 \mathrm{~V}(v s . \mathrm{Ag} / \mathrm{AgCl})$ 。图 6(e)为 $\mathrm{BVO} / 5 \mathrm{BFO}$ 在无偏压下的光电流密度, 稳定在 $0.7 \mathrm{~mA} \cdot \mathrm{cm}^{-2}$ 左右, 说明制备的样品具有良好的循 环稳定性。

\section{6 外场极化下的光电化学性能分析}

图 7 为外场极化处理前后 $\mathrm{BVO} / 5 \mathrm{BFO}$ 的 $I-t$ 曲 线与线性伏安曲线( $v s . \mathrm{NHE})$ 。正极化可以显著提升 $\mathrm{BVO} / 5 \mathrm{BFO}$ 复合物的光电化学性能, $\mathrm{BVO} / 5 \mathrm{BFO}$ 经 正极化后在外加偏压为 $0 \mathrm{~V}$ (vs. $\mathrm{Ag} / \mathrm{AgCl})$ 时的光电 流密度 $\left(0.91 \mathrm{~mA} \cdot \mathrm{cm}^{-2}\right)$ 高于未经极化的 $\mathrm{BVO} / 5 \mathrm{BFO}$ $\left(0.72 \mathrm{~mA} \cdot \mathrm{cm}^{-2}\right)$, 比纯 $\mathrm{BVO}\left(0.43 \mathrm{~mA} \cdot \mathrm{cm}^{-2}\right)$ 提升 1 倍 以上。负极化则抑制了 $\mathrm{BVO} / 5 \mathrm{BFO}$ 的光电化学性能, 样品的光电流密度在负极化处理后明显降低。外场 极化对铁电材料光电化学性能的影响可以归因于其 对铁电复合材料界面处能带弯曲与耗尽层厚度的影 响 ${ }^{[8]}$, 进而影响光生载流子的传输。

\section{7 异质结机理分析}

图 8 为 $\mathrm{BVO} / n \mathrm{BFO}$ 复合材料的异质结能级结构 示意图。光照时 $\mathrm{BVO} / n \mathrm{BFO}$ 复合物价带上产生的光 生电子跃迁到导带上, 由于 $\mathrm{BiFeO}_{3}$ 的导带位置较高,
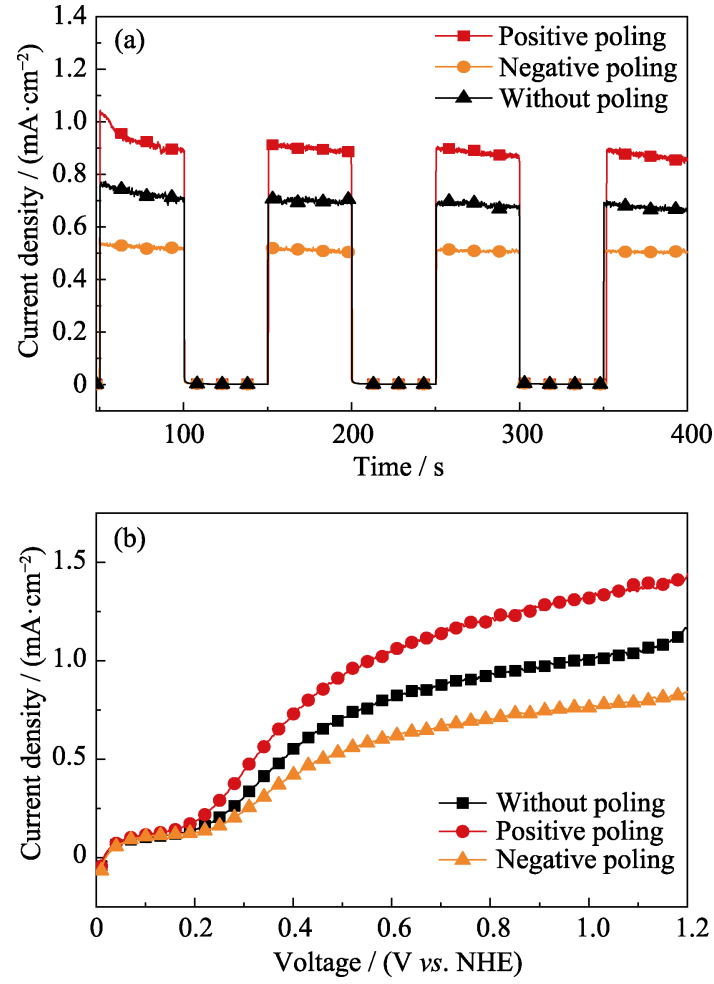

图 $7 \mathrm{BVO} / 5 \mathrm{BFO}$ 极化前后的(a) $I-t$ 曲线和(b)线性伏安曲线 Fig. 7 (a) I- $t$ plots and (b) linear sweep voltammograms of $\mathrm{BVO} / 5 \mathrm{BFO}$ before and after polarization

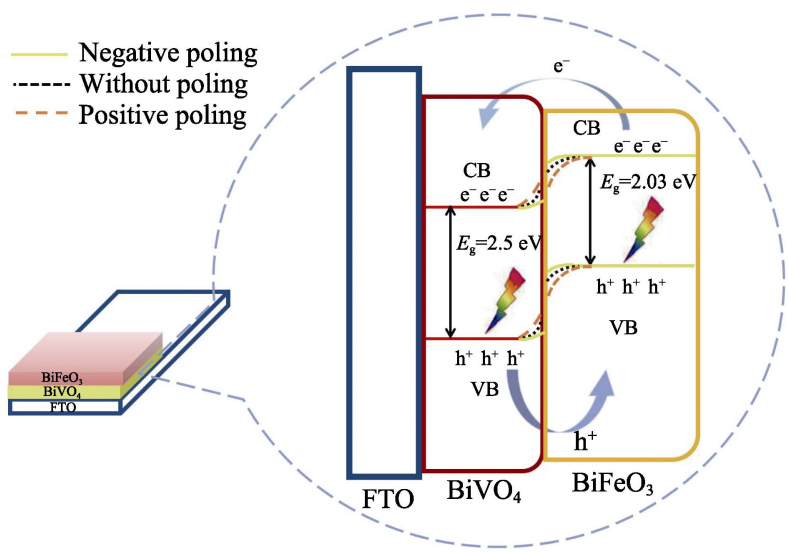

图 $8 \mathrm{BVO} / n \mathrm{BFO}$ 复合物的能带结构示意图

Fig. 8 Schematic diagram of $\mathrm{BVO} / n \mathrm{BFO}$ band energy

光照产生的光生电子由 $\mathrm{BiFeO}_{3}$ 导带转移到 $\mathrm{BiVO}_{4}$ 的导带。同时由于 $\mathrm{BiVO}_{4}$ 的价带位置较低, $\mathrm{BiVO}_{4}$ 价带上产生的光生空穴将转移到 $\mathrm{BiFeO}_{3}$ 的价带, 因 此光生电子与空穴得以有效分离, 延长了光生载流 子的寿命。由于 $\mathrm{BiVO}_{4}$ 与 $\mathrm{BiFeO}_{3}$ 属于铁电材料, 其 内部的自发极化与外场极化会改变界面处能带弯曲 的程度, 影响光生电荷的转移 ${ }^{[18-20]}$ 。外场正极化后, 在 $\mathrm{BiVO}_{4}$ 与 $\mathrm{BiFeO}_{3}$ 界面处 $\mathrm{BiFeO}_{3}$ 能带向下弯曲程 度增大, $\mathrm{BiVO}_{4}$ 能带向上弯曲程度增大，导致耗尽 层宽度增大，有利于光生载流子的传输，进而提升 光电化学性能。外场负极化后, 耗尽层宽度减小, 
$\mathrm{BiVO}_{4}$ 与 $\mathrm{BiFeO}_{3}$ 界面处能带弯曲程度减小, 阻碍了 光生载流子的传输 ${ }^{[11]}$ 。

\section{3 结论}

通过旋涂法, 采用 $\mathrm{BiFeO}_{3}$ 对 $\mathrm{BiVO}_{4}$ 薄膜进行 修饰, 获得的 $\mathrm{BVO} / n \mathrm{BFO}$ 薄膜界面处形成了异质结, 可以有效地抑制光生电子一空穴的复合。铁电材料外 场极化处理造成的能带弯曲使光生电荷加速转移, 导致 $\mathrm{BVO} / 5 \mathrm{BFO}$ 复合物具有较小的电化学阻抗和 较大的光响应电流密度, 最大光电流密度为 $0.91 \mathrm{~mA} \cdot \mathrm{cm}^{-2}$, 比未修饰试样 $\left(0.43 \mathrm{~mA} \cdot \mathrm{cm}^{-2}\right)$ 提升 1 倍以上。经过 $\mathrm{BFO}$ 修饰后的 $\mathrm{BVO} / n \mathrm{BFO}$ 复合物的 光电化学性能显著提高, 在光催化制氢以及光降解 等领域具有广泛的应用前景。

\section{参考文献:}

[1] FAN T, CHEN C C, TANG Z H. Hydrothermal synthesis of novel $\mathrm{BiFeO}_{3} / \mathrm{BiVO}_{4}$ heterojunctions with enhanced photocatalytic activities under visible light irradiation. RSC Advances, 2016, 6(12): 9994-10000.

[2] LUO W J, YANG Z S, ZHANG J Y, et al. Solar hydrogen generation from seawater with a modified $\mathrm{BiVO}_{4}$ photoanode. Energy \& Environmental Science, 2011, 4(10): 4046-4046.

[3] WANG S C, CHEN P, BAI Y, et al. New $\mathrm{BiVO}_{4}$ dual photoanodes with enriched oxygen vacancies for efficient solar-driven water splitting. Adv. Mater, 2018, 30(20): e1800486.

[4] YANG T J, WANG S L, YANG H G, et al. Preparation of $\mathrm{BiVO}_{4}$ by hydrothermal method and its photocatalytic properties under visible light irradiation. Journal of Yulin Normal University, 2018, 39(5): 55-64.

[5] ZHOU L, WANG W Z, LIU S W, et al. A sonochemical route to visible-light-driven high-activity $\mathrm{BiVO}_{4}$ photocatalyst. Journal of Molecular Catalysis A: Chemical, 2006, 252(1/2): 120-124.

[6] YU J Q, KUDO A. Hydrothermal synthesis of nanofibrous bismuth vanadate. Chemistry Letters, 2005, 34(6): 850-851.

[7] ZHANG K, JIN B J, PARK C, et al. Black phosphorene as a hole extraction layer boosting solar water splitting of oxygen evolution catalysts. Nature Communications, 2019, 10: 2001-2010.

[8] YANG W G, YU Y H, STARR M, et al. Ferroelectric polarizationenhanced photoelectrochemical water splitting in $\mathrm{TiO}_{2}-\mathrm{BaTiO}_{3}$ core-shell nanowire photoanodes. Nano Lett., 2015, 15(11): 7574-7580.

[9] LEE H, JOO H Y, YOON C, et al. Ferroelectric $\mathrm{BiFeO}_{3} / \mathrm{TiO}_{2}$ nanotube heterostructures for enhanced photoelectrochemical performance. Current Applied Physics, 2017, 17(5): 679-683.

[10] WANG Y Z, CHEN D, WANG S, et al. Photoassisted electrodeposition of cobalt-phosphate cocatalyst on $\mathrm{BiFeO}_{3}$ thin film photoanode for highly efficient photoelectrochemical performances of water oxidation. Journal of The Electrochemical Society, 2019, 166(8): D308-D314.

[11] XIE J L, GUO C X, YANG P P, et al. Bi-functional ferroelectric $\mathrm{BiFeO}_{3}$ passivated $\mathrm{BiVO}_{4}$ photoanode for efficient and stable solar water oxidation. Nano Energy, 2017, 31: 28-36.

[12] KIM T W, CHOI K S. Nanoporous $\mathrm{BiVO}_{4}$ photoanodes with dual-layer oxygen evolution catalysts for solar water splitting. Science, 2014, 343(6174): 990-994.

[13] CHEN Y, ZHOU K C, HUANG S P, et al. Hydrothermal synthesis and photocatalytic property of $\mathrm{BiVO}_{4}$ nanosheets. The Chinese Journal of Nonferrous Metals, 2011, 21(7): 1570-1579.

[14] LI C J, ZHANG P, LÜ R, et al. Selective deposition of $\mathrm{Ag}_{3} \mathrm{PO}_{4}$ on monoclinic $\mathrm{BiVO}_{4}(040)$ for highly efficient photocatalysis. Small, 2013, 9(23): 3950-3956.

[15] GONG S Q, JIANG Z J, SHI P H, et al. Noble-metal-free heterostructure for efficient hydrogen evolution in visible region: molybdenum nitride/ultrathin graphitic carbon nitride. Applied $\mathrm{Ca}$ talysis B: Environmental, 2018, 238: 318-327.

[16] YU L Q, HUANG C X, ZHANG Y P, et al. Photoelectrochemical properties of $\mathrm{MoS}_{2}$ modified $\mathrm{TiO}_{2}$ nanotube arrays. Journal of Inorganic Materials, 2016, 31(11): 1237-1241.

[17] ZHANG Y P, GU R, ZHENG S, et al. Long-life Li-S batteries based on enabling the immobilization and catalytic conversion of polysulfides. J. Mater. Chem. A, 2019, 7(38): 21747-21758.

[18] SONG J, KIM T L, LEE J, et al. Domain-engineered $\mathrm{BiFeO}_{3}$ thin-film photoanodes for highly enhanced ferroelectric solar water splitting. Nano Research, 2018, 11(2): 642-655.

[19] SHI J, ZHAO P. Piezoelectric polarization enhanced photovoltaic performance in depleted-heterojunction quantum-dot solar cells. Adv. Mater., 2013, 25(6): 916-921.

[20] SANG Y J, SONG J, LEE S. Photoelectrochemical device designs toward practical solar water splitting: a review on the recent progress of $\mathrm{BiVO}_{4}$ and $\mathrm{BiFeO}_{3}$ photoanodes. Appl. Sci., 2018, 8(8): 1388 . 